\title{
Revistas científicas editadas por universidades en Web of Science: características y contribución a la marca universidad
}

\author{
Scientific journals published by universities in Web \\ of Science: Characteristics and contribution to the \\ university brand
}

\author{
Rafael Repiso; Enrique Orduña-Malea; Ignacio Aguaded
}

Cómo citar este artículo:

Repiso, Rafael; Orduña-Malea, Enrique; Aguaded, Ignacio (2019). “Revistas científicas editadas por universidades en Web of Science: características y contribución a la marca universidad". El profesional de la información, v. 28, n. 4, e280405.

https://doi.org/10.3145/epi.2019.jul.05

Artículo recibido el 04-01-2019

Aceptación definitiva: 25-03-2019

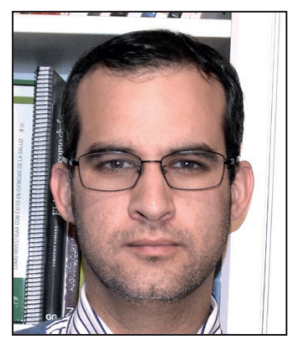

Rafael Repiso $\bowtie$

https://orcid.org/0000-0002-2803-7505

Universidad Internacional de La Rioja

Av. de la Paz, 137.

26006 Logroño, España

rafael.repiso@gmail.com

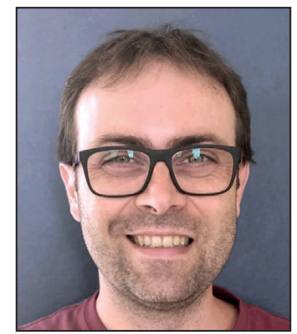

Enrique Orduña-Malea

http://orcid.org/0000-0002-1989-8477

Universitat Politècnica de València

Camino de Vera, $\mathrm{s} / \mathrm{n}$.

46020 Valencia, España

enorma@upv.es

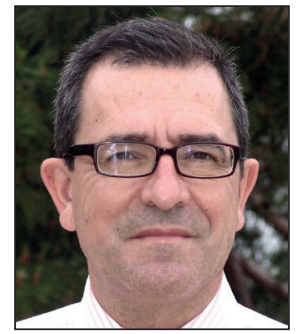

Ignacio Aguaded

https://orcid.org/0000-0002-0229-1118

Universidad de Huelva

Campus de El Carmen

Avda. Tres de Marzo, s/n.

21071 Huelva, España

aguaded@uhu.es

\section{Resumen}

El principal objetivo del presente trabajo es analizar la aportación que las revistas académicas hacen a la marca de la universidad. Para ello se identifica la presencia de revistas y universidades editoras en las bases de datos bibliográficas de referencia (Science Citation Index, Social Sciences Citation Index y Arts \& Humanities Citation Index, de la Web of Science) y se realiza un análisis bibliométrico para describir la población de revistas estudiadas, analizando elementos como la distribución por países, universidades editoras, coedición, tasas de éxito de indexación, presencia por áreas e impacto científico. Se identifican finalmente 1.492 revistas editadas por 387 universidades de 56 países. Las universidades anglosajonas destacan por su elevado número de revistas indexadas en WoS, especialmente Oxford y Cambridge, que juntas agrupan el $38,7 \%$ de las cabeceras. 


\title{
Palabras clave
}

Revistas científicas; Comunicación académica; Universidades; Marca universidad; Web of Science; Revistas universitarias; Edición científica; Bibliometría.

\begin{abstract}
This work aims to analyse the contribution that academic journals make to their university brand. To this end, the presence of journals and university in the reference databases (Science Citation Index, Social Sciences Citation Index and Arts \& Humanities Citation Index, part of Web of Science) are identified. Later, a bibliometric analysis is carried out to describe the population of journals studied, analysing elements such as distribution by country, publishing universities, co-publishing, indexing success rates, presence by area and scientific impact. Finally 1,492 journals published by 387 universities in 56 countries are identified. Anglo-Saxon universities stand out for their high number of journals indexed in WoS, especially Oxford and Cambridge, which together account for $38.7 \%$ of the titles.
\end{abstract}

\section{Keywords}

Scientific journals; Scholarly communication; Universities; University brand; Web of Science; University journals; Academic journals; Scientific edition; Bibliometrics.

\section{Introducción}

Las universidades son sistemas complejos que trabajan en torno a un intangible diferencial, el conocimiento. La universidad es una institución que funciona adquiriendo, generando y transmitiendo conocimiento. La adquisición se centra en la compra de fondos documentales, suscripción a fuentes informativas, tareas de evaluación y contratación de profesores. La transferencia es la transformación de conocimientos y habilidades especializadas en nuevos productos o servicios a la sociedad en sus múltiples dimensiones. La generación de conocimiento recae en la dimensión de investigación (Orduña-Malea, 2012), en la que el profesor universitario se erige como principal dinamizador del mismo.

No obstante, en el desarrollo de una investigación se necesitan elementos externos que validen y difundan los trabajos internos. Este papel es protagonizado por las revistas científicas. En ese sentido, algunas universidades son gestoras de capital intelectual de primer orden; sus editoriales universitarias y revistas constituyen elementos fundamentales en la producción y difusión de conocimiento, así como uno de los principales mecanismos para medir su impacto.

Las revistas científicas nacieron de la mano de sociedades, es decir no son originalmente universitarias. Es la integración de la investigación como elemento de la universidad en el siglo XIX lo que estimula la creación de revistas editadas, aun concentrando su energía editorial en monográficos (Clement, 2011; Hayes; Holley, 2014). Por tanto no son exclusividad universitaria, sino que las universidades son unas editoras más. De hecho, una de las preguntas que pretende responder este trabajo es qué peso tienen las revistas editadas por universidades en la producción científica mundial. Para ello es importante reflexionar sobre las ventajas y desventajas que supone la edición académica.

La hipótesis de este trabajo es que las revistas son un buen indicador para medir la calidad de una universidad. La investigación y generación de capital intelectual es un elemento clave y diferenciador en la industria y en los entornos competitivos modernos (Cuganesan; Petty; Finch, 2006). En el contexto universitario es la producción científica el elemento utilizado para caracterizar a la universidad y sus profesores, departamentos, etc. Es indiscutible la importancia que en todo este proceso tienen las revistas, exigiendo repensar los beneficios que las universidades obtienen de ellas. La capacidad de una universidad para crear, gestionar y mantener revistas científicas de impacto en bases de datos internacionales es un criterio sencillo y justificado que refleja su calidad en materia de dimensión editorial y referente científico, y que podría ser utilizado como parámetro de calidad en los rankings universitarios.

Para tener una revista científica de forma autónoma se necesita soporte editorial y recursos humanos (masa crítica de investigadores especializados)

¿Qué se necesita para tener una revista científica de forma autónoma?: Soporte editorial y recursos humanos (masa crítica de investigadores especializados). Por tanto, para editar, un centro debe poseer suficiente número de investigadores que destaquen en el área temática desarrollada, o tener al menos capacidad para atraerlos.

Actualmente, si excluimos modelos minoritarios (asociaciones de toda índole, colegios profesionales, fundaciones, entidades gubernamentales, etc.), los principales modelos de editoriales de revistas son:

- de asociaciones científicas;

- de universidades;

- de centros de investigación;

- editoriales científicas privadas.

En los tres primeros casos el servicio de publicación (y por tanto la edición) es un objetivo secundario, pues cuentan con su propia plantilla de personal científico productor de investigación. En cambio, en las editoriales científicas privadas la 
edición y difusión científica sí es una prioridad, sin contar con recursos científicos propios.

"La verdadera distinción entre los editores comerciales y los que no tienen ánimo de lucro radica en sus objetivos: el lucro del accionista frente a una misión cultural multifacética" (Esposito, 2007).

Los fines de las editoriales de revistas científicas varían según modelos. Para la industria editorial el principal factor es el económico. Para las sociedades científicas y universidades es el teleológico. La descripción que en su sitio web hace la Oxford University Press de sus fines vinculados a los de la universidad no dejan lugar a duda:

"Oxford University Press es un departamento de la University of Oxford. Su finalidad es promover el objetivo de Excelencia de la universidad en investigación, becas y educación" (Oxford University, 2018).

Por tanto, las editoriales universitarias y sus revistas editadas son elementos que ayudan al cumplimiento de los objetivos científicos de las instituciones, visibilizando la presencia prioritaria en un área de conocimiento de la universidad a nivel global.

¿Qué aporta una revista científica a una universidad?

a) Ventajas docentes

- Escuelas de formación en comunicación científica: las universidades aproximan alumnos de postgrado y profesores a la investigación facilitando la colaboración en revistas del centro.

b) Ventajas científicas

- Difusoras de conocimiento: las revistas universitarias publican investigación original validada por la universidad con recursos externos: trabajos realizados por investigadores de instituciones externas.

- Hub científico: las revistas científicas estructuran la comunidad científica de un área, pues sus investigadores participan en ellas como lectores, evaluadores y autores. Por tanto, se convierten en un referente en tanto en cuanto éstas se posicionan.

c) Ventajas estructurales

- Revistas como gatekeepers de la profesión: han sido definidas como las gatekeepers de la ciencia (Crane, 1967), pues sus funciones son evaluar y filtrar las malas investigaciones. Regazzi (2015) reflexiona sobre el poder que tienen para consolidar la carrera de los investigadores, cuando en los sistemas académicos la progresión académica se basa en la evaluación de méritos científicos. Las revistas, al menos las relevantes, tienen el poder indirecto de promocionar a los miembros de la comunidad, aunque según Furlough (2008), las universidades están perdiendo esta capacidad de evaluar la ciencia en favor de otros agentes.

- Revistas como rédito económico: la edición de revistas universitarias reduce la dependencia de las editoriales comerciales (Corera-Álvarez; Molina-Molina, 2016). Además, las universidades pueden obtener ingresos por la venta y acceso a sus revistas. Aunque sólo las mejores revistas están en disposición de rentabilizarse.

- Revistas como valor de intercambio:

"El canje de revistas científicas ha sido utilizado tradicionalmente por las bibliotecas universitarias y científicas como un medio de enriquecer sus colecciones" (Delgado-López-Cózar; Ruiz-Pérez; Jiménez-Contreras, 2006).

Proyectos como MUSE, que en principio fomentaba la digitalización de revistas universitarias y su acceso (Lewis, 1995), aglutinan a editores universitarios, dan a conocer sus revistas y permiten obtener rédito de ellas. http://muse.jhu.edu

- Revistas como nexo relacional con otras instituciones: se pueden aprovechar las ventajas estructurales de las revistas para poner al servicio de la institución su capital social fomentando y dinamizando la colaboración entre investigadores e instituciones.

Bajo la 'marca universidad' podemos valorar el significado en su conjunto de la edición de revistas para una universidad, los beneficios que reporta en el cumplimiento de los objetivos y cómo ésta se visibiliza internacionalmente. La edición de revistas refleja la calidad, prestigio y excelencia en un ámbito científico concreto, incorporando una ventaja extraordinaria: la institución editora no genera conocimiento, sino que lo evalúa por parte del editor otorgándole validez, registrándolo, difundiéndolo y agregando su propio sello.

El estudio de la universidad desde el paradigma de la marca no tiene más de dos décadas. Con anterioridad se habían estudiado ideas como el prestigio, la reputación o la imagen. Sin embargo, la marca va más allá y parte de dos dimensiones: la que el centro quiere transmitir y la que percibe el público (Aaker; Álvarez-del-Blanco, 2014). La marca se sitúa en relación directa con las metas y fines de la institución y la realidad de la misma (Aaker; Joachimsthaler, 2000). Según Ali-Choudhury, Bennett y Savani (2009) existen tres áreas con diferentes dimensiones de la universidad: elementos identificativos, naturaleza e imaginario asociado (figura 1).

La marca global de la universidad se ve reforzada por las individuales de sus componentes, profesores, facultades, departamentos o revistas beneficiándose en un prestigio mutuo. Las revistas académicas se encontrarían dentro del apartado "Producción científica" y cada una de ellas sería una marca diferente dentro de la cartera que gestiona la universidad. 


\begin{tabular}{|c|c|c|c|c|c|c|c|}
\hline \multirow{2}{*}{$\begin{array}{c}\text { Elementos } \\
\text { identificativos }\end{array}$} & Nombre & \multicolumn{3}{|c|}{ Logotipos } & Lemas & Escudos & Acrónimos \\
\hline & \multicolumn{7}{|c|}{ Otros/Merchandising } \\
\hline \multirow{5}{*}{$\begin{array}{c}\text { Naturaleza de las } \\
\text { universidades }\end{array}$} & Historia & \multicolumn{3}{|c|}{ Localización } & Sedes & \multicolumn{2}{|c|}{ Producción científica } \\
\hline & \multicolumn{5}{|c|}{ Orientación docencia/investigación } & \multicolumn{2}{|c|}{ Recursos humanos } \\
\hline & \multicolumn{4}{|c|}{ Acreditación Web } & Propiedad & \multicolumn{2}{|c|}{ Especialización } \\
\hline & \multicolumn{3}{|c|}{ Modalidad docente } & \multicolumn{3}{|c|}{ Oferta deportiva } & Tamaño \\
\hline & \multicolumn{3}{|c|}{ Tipo de admisión } & & rta cultural & \multicolumn{2}{|c|}{ Infraestructuras } \\
\hline \multirow{2}{*}{$\begin{array}{l}\text { Imaginario asociado } \\
\text { con la universidad }\end{array}$} & \multicolumn{5}{|c|}{ Características de las carreras } & \multirow{2}{*}{\multicolumn{2}{|c|}{ Vida académica }} \\
\hline & \multicolumn{5}{|c|}{ Empleo futuro } & & \\
\hline
\end{tabular}

Figura 1. Dimensiones de la marca universidad

Nota: clasificación ampliada de las dimensiones de la marca universidad propuestas por Ali-Choudhury, Bennett y Savani (2009). La Web no estaba incluida en la clasificación original.

De entre el público general y académico, las revistas tienen una mayor incidencia en el segundo. No obstante, también las revistas alcanzan al público general cuando sus investigaciones transcienden a los medios de masas (Repiso; Merino-Arribas; Chaparro-Domínguez, 2016; Repiso; Chaparro-Domínguez, 2018). Un ejemplo de cómo asociar la marca de una revista beneficia a una institución es la coedición en árabe de la revista Nature que realizan desde 2012 King Abdulaziz City for Science and Technology (Kacst) y Macmillan Scientific Communications (MSC), partner exclusivo de Nature Publishing Group (NPG).

El objetivo de este trabajo es identificar y caracterizar las revistas editadas por universidades que se sitúan como referentes mundiales, entendiendo por referentes aquellas con presencia en las bases de datos Science Citation Index (SCI), Social Sciences Citation Index (SSCI) y Arts \& Humanities Citation Index (A\&HCI).

Adicionalmente se plantean las siguientes preguntas de investigación:

1: ¿Cuáles son las universidades que editan una mayor cantidad de revistas de referencia?

2: ¿Qué sistemas universitarios, por países, están liderando la edición científica?

3: ¿Qué peso (cobertura) e impacto (cuartil en los $J C R$ ) tienen las revistas editadas por universidades en el contexto de las revistas que lideran la investigación internacional? ¿Existen diferencias entre las distintas áreas de conocimiento?

\section{Metodología}

Para dar respuesta a las preguntas de investigación se han identificado todas las revistas científicas editadas por universidades en las tres bases de datos core de Web of Science; Science Citation Index, Social Sciences Citation Index y Arts \& Humanities Citation Index.

"Estos índices cubren tres grupos principales de disciplinas: Artes y Humanidades, Ciencias y Ciencias Sociales" (Levine-Clark; Gil, 2008).

La elección de estas bases de datos se fundamenta en que en estos productos

"se encuentra la bibliografía científica de mayor difusión, visibilidad e impacto internacional" (Delgado-López-Cózar; Fernández-Cano, 2002),

“mayor cobertura temporal y más fácil accesibilidad en el mundo académico” (Zhang; Leung, 2015).

Emerging Sources Citation Index ha sido excluida del estudio porque recopila revistas no referentes en proceso de evaluación a la espera de incluirse en las citadas bases de datos élite (Repiso; Torres-Salinas, 2016).

La identificación de las revistas partió de una primera fase en la que se revisaron todas aquellas indexadas en la Master Journal List de Web of Science, identificando publicaciones en las que aparecía el nombre de una universidad en la dirección de correspondencia o en el propio. Junto al nombre de la revista se recopiló toda la información descriptiva de cada publicación (URL, ISBN, universidad editora, etc.). Este proceso se realizó en abril de 2017.

http://mil.clarivate.com

Aunque la gran mayoría de universidades incluyen el término 'universidad' en su nombre ('University of...'), existe un reducido grupo (como las universidades politécnicas con el MIT a la cabeza) que no lo incluyen. Para validar las revistas creadas por este grupo, se recopiló un listado exhaustivo de universidades que cumpliesen esta característica a partir del 
Ranking Web de Universidades del Mundo, para buscar posteriormente su presencia en la Master Journal List de WoS. La elección de este ranking se debió a que es el más exhaustivo cubriendo más de 27.000 instituciones de educación superior.

http://webometrics.info

Una segunda fase de validación consistió en visitar el sitio web de estas revistas, considerado ya desde hace unos años como la fuente primaria frente a la revista en papel (Carpenter, 2008), y comprobar que eran editadas en la actualidad por universidades. En esta identificación individual también se registraron dos datos:

- si aparecía el nombre o un logotipo de la universidad editora en sus páginas principales;

- si la revista era coeditada por más de una institución.

Una vez identificadas y verificadas en WoS, se realizó un análisis general de su presencia en las bases de datos seleccionadas $(S C l, S S C l$ y $A \& H C l$ ) así como su impacto medido en cuartiles y su distribución por países y universidades editoras, estudiándose las universidades que editan un mayor número de revistas (por encima de ocho). Todos los datos fueron exportados a una hoja de cálculo para su análisis. Adicionalmente se utilizó el diagrama de Sankey (figura 3) mediante SankeyMatic (Bogart, 2017).

\section{Resultados}

Se han localizado 1.492 revistas editadas por 387 universidades de 56 países, de las cuales el 81,2\% (1.211) identifican en portada su pertenencia a una universidad a través de imágenes y enlaces. En cambio, un reducido número (256) no identifican este hecho, empleando páginas secundarias del sitio web ("address" o "about"). En

La presencia de las revistas editadas por universidades en los Journal Citation Reports (JCR) se agrupa en los últimos cuartiles el resto de publicaciones (25) no se ha logrado localizar la web, aunque son identificadas por WoS y Ulrichs como revistas editadas por universidades.

Las revistas editadas por universidades representan una pequeña parte del total de revistas indexadas en $\mathrm{SCl}$, $\mathrm{SSCl}$ y A\&HCl de Web of Science (figura 2). Apenas un 11,6\% del total (en el momento del análisis se registraban 12.860 revistas), pero su presencia es desigual según las bases de datos. Representan:

- 6,3\% en Science Citation Index;

- 14,6\% en Social Sciences Citation Index;

- 33,8\% en Arts \& Humanities Citation Index.

De igual manera, la presencia de las revistas editadas por universidades en los Journal Citation Reports (JCR) se agrupa en los últimos cuartiles, siendo el cuarto cuartil (Q4) el que agrupa más revistas universitarias, y el primero (Q1) el que presenta un menor número.

Las universidades de Gran Bretaña, Estados Unidos y Canadá agrupan un mayor número de revistas editadas (figura 3). De las 211 revistas editadas por universidades incluidas en el Q1 de SCI y SSCl, hasta 164 (77,7\%) corresponden a Gran Bretaña mientras que 41 publicaciones $(19,4 \%)$ pertenecen a Estados Unidos. En el caso del Q2 el patrón es similar, Reino Unido reúne al $59,4 \%$ de las revistas y Estados Unidos al 24\% (52 revistas). Por tanto, la mayor parte de las revistas del Q1 (97\%) son editadas por universidades británicas y estadounidenses. En el Q2 sucede algo similar, mientras que las universidades de los restantes países suelen posicionarse en Q3 y Q4.

Las revistas editadas por universidades tienden a acumularse en unas pocas instituciones, los 20 centros con más revistas agrupan el 61,9\% del total (tabla 1). Las dos universidades con mayor número de revistas en WoS agrupan el 38,7\% de las cabeceras, Oxford $(19,6 \%)$ y Cambridge University (19,1\%). La mayor parte de las universidades sólo tienen una revista en los índices de WoS (258 universidades).

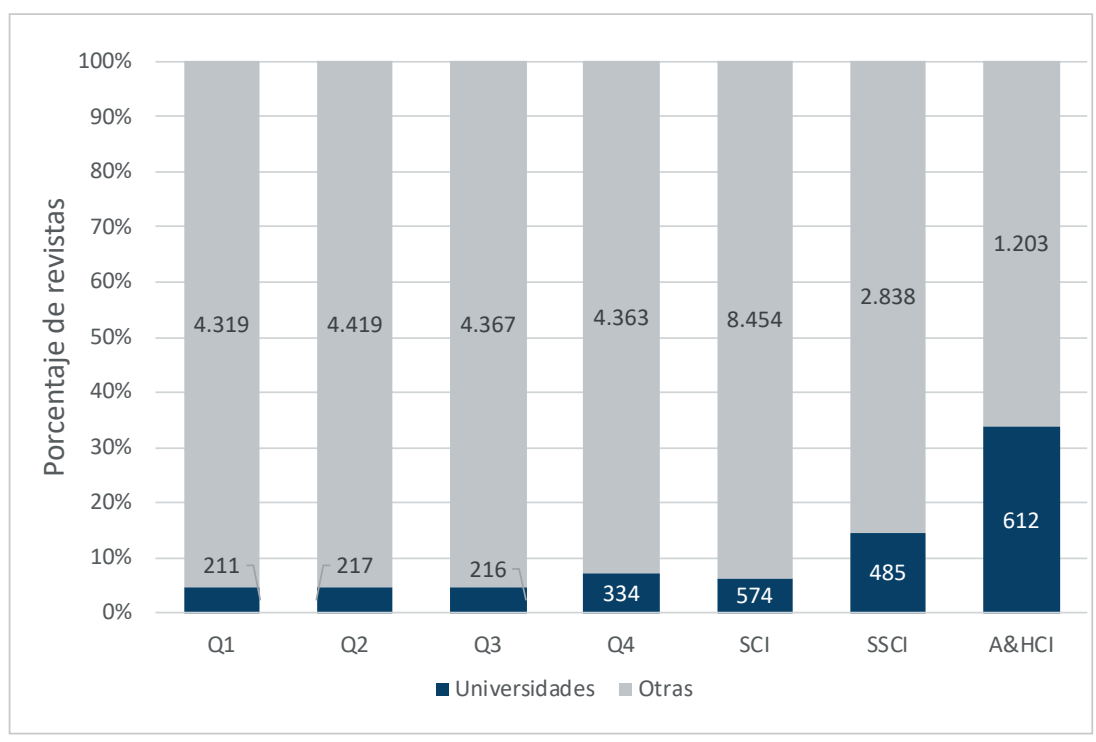

Figura 2. Distribución de revistas editadas por universidades en Web of Science según base de datos $(\mathrm{SCl}, \mathrm{SSCl}, \mathrm{A \& HCl})$ y cuartiles 


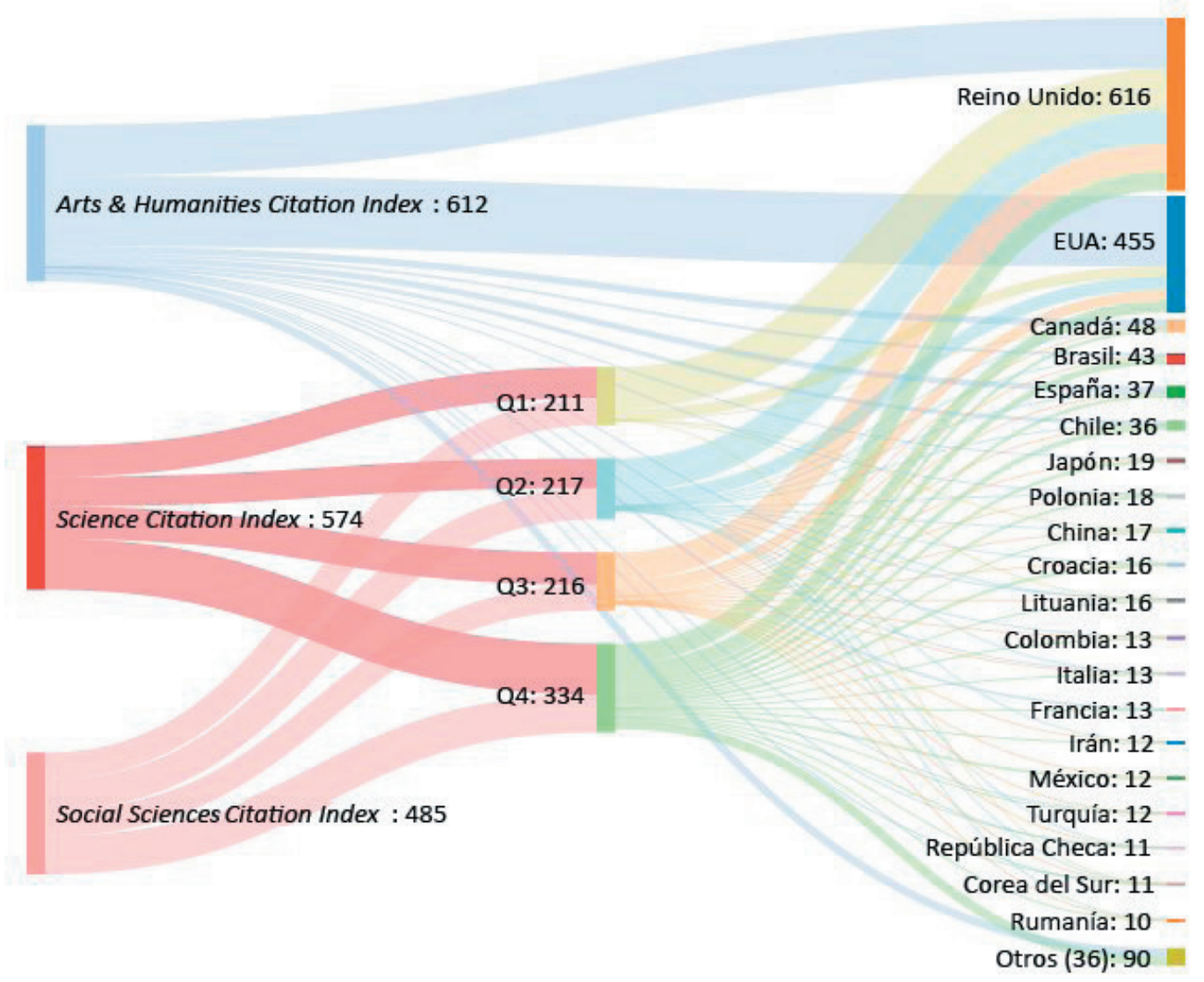

Figura 3. Distribución de revistas editadas por universidades en Web of Science (WoS) según bases de datos (SCI, SSCI, A\&HCl), cuartiles y países (2018)

Tabla 1. Editoriales universitarias en Web of Science según número de revistas en 2018 (Top 20, universidades que publican 8 ó más revistas)

\begin{tabular}{|c|c|c|c|c|c|c|c|c|c|}
\hline $\mathbf{R}$ & Universidades & $\begin{array}{l}\text { Total } \\
\text { WoS }\end{array}$ & Coed. & $\mathrm{SCl}$ & SSCI & Q1 & $A \& H C I$ & $\begin{array}{c}\text { Total } \\
\text { revistas }\end{array}$ & $\begin{array}{l}\text { Tasa } \\
\text { éxito }\end{array}$ \\
\hline 1 & University of Oxford & 292 & 195 & 162 & 114 & 113 & 59 & 433 & 67,4 \\
\hline 2 & University of Cambridge & 285 & 136 & 113 & 121 & 49 & 108 & 412 & 69,2 \\
\hline 3 & University of Chicago & 54 & 21 & 11 & 31 & 13 & 20 & 75 & 72,0 \\
\hline 4 & Johns Hopkins University & 57 & 23 & 4 & 14 & 1 & 46 & 91 & 62,6 \\
\hline 5 & Duke University & 34 & 13 & 4 & 12 & 4 & 27 & 56 & 60,7 \\
\hline 6 & Massachusetts Institute of Technology & 24 & 11 & 7 & 9 & 2 & 11 & 36 & 66,6 \\
\hline 7 & University of California & 22 & 1 & 2 & 6 & 2 & 18 & 55 & 40,0 \\
\hline 8 & University of Edinburgh & 20 & 6 & 2 & 5 & 1 & 19 & 45 & 44,4 \\
\hline 9 & University of Toronto & 16 & 1 & 3 & 5 & 0 & 8 & 45 & 35,5 \\
\hline 10 & University of Zagreb & 14 & 0 & 10 & 4 & 0 & 0 & *14 & 100 \\
\hline 11 & Penn State University & 14 & 5 & 0 & 1 & 0 & 13 & 54 & 29,6 \\
\hline 12 & University of Illinois & 13 & 2 & 0 & 2 & 0 & 11 & 40 & 32,5 \\
\hline 13 & Universidade de São Paulo & 12 & 0 & 10 & 4 & 0 & 0 & 177 & 6,8 \\
\hline 14 & Harvard University & 11 & 0 & 0 & 9 & 3 & 2 & *87 & 12,6 \\
\hline 15 & Indiana University & 11 & 3 & 1 & 1 & 1 & 9 & 47 & 23,4 \\
\hline 16 & Pontificia Universidad Católica de Chile & 11 & 0 & 3 & 4 & 0 & 6 & 33 & 33,3 \\
\hline 17 & University of Pennsylvania & 11 & 1 & 0 & 3 & 0 & 8 & 19 & 57,9 \\
\hline 18 & University of Hawaii & 8 & 4 & 1 & 2 & 1 & 5 & 25 & 32,0 \\
\hline 19 & University of Wisconsin & 8 & 1 & 0 & 4 & 1 & 4 & 12 & 66,7 \\
\hline 20 & Vilnius Gediminas Technical University & 8 & 0 & 5 & 3 & 0 & 0 & 16 & 50,0 \\
\hline
\end{tabular}

* Los datos han sido extraídos de la base de datos Ulrichs.

Coed.: número de las revistas WoS editadas en colaboración con otra institución.

Total revistas: número de revistas académicas de cada universidad incluyendo aquellas que no están indexadas en WoS:

Tasa de éxito: porcentaje de revistas incluidas en WoS respecto del total de revistas editadas. 
Las universidades con mayor concentración de revistas son anglosajonas a excepción de:

- Sveučilišta u Zagrebu / University of Zagreb (Croacia);

- Universidade de São Paulo / University of São Paulo (Brasil);

- Pontificia Universidad Católica de Chile (Chile);

- Vilnius Gediminas Technical University (Lituania).

Un aspecto destacado de editoriales universitarias de éxito es que muchas de sus revistas tienen coedición. La University of Oxford tiene un 66,7\% de revistas indexadas en Web of Science coeditadas con otras instituciones: asociaciones y organismos internacionales de prestigio internacional como la Royal Astronomical Society o

En el contexto universitario, Reino Unido es el país con mayor número de revistas en WoS (616) seguido de Estados Unidos (455) el Banco Mundial. Algo parecido, pero en menor medida, sucede con las revistas de Cambridge (47,7\%), University of Chicago (38\%) o Johns Hopkins University (40,3\%).

También destaca su tasa de éxito, entendiendo el éxito como el situar a sus revistas en los índices más prestigiosos del mundo. En este aspecto la University of Zagreb es la institución que tiene una editorial con el porcentaje de revistas indexadas más alto, pues todas ellas se encuentran en WoS. En contraste, la Universidade de São Paulo sólo tiene indexadas el 6,8\% (12) de sus 177 revistas.

\section{Discusión y conclusiones}

Sólo 387 universidades en el mundo tienen revistas indexadas en las bases de datos élite de Web of Science (SCI, SSCI y $A \& H C l)$. Aunque resulta habitual que las revistas universitarias identifiquen a su institución visiblemente en la Web, compartiendo su prestigio con el de la revista y viceversa, un grupo reducido de revistas (256) oculta esta información en páginas secundarias de poco tránsito para el lector habitual. Las revistas como "productos" de las universidades, además de utilizar recursos de estas instituciones para alcanzar sus fines, deberían utilizar el respaldo de su universidad y, en contrapartida, situar sus resultados bajo el paraguas de la entidad editora, al objeto de que ambos actores se beneficien.

\section{RQ 1: Universidades editoras}

Al igual que en el mundo de la edición de revistas en general, donde unas pocas editoriales comerciales, Reed-Elsevier, Wiley-Blackwell, Taylor \& Francis, Sage, Springer y Wolters Kluwer controlan alrededor del 60\% de la industria (Shapiro, 2013), una serie de universidades (Oxford, Cambridge, Chicago...) destacan como editoras de las principales revistas de impacto. Destaca por ejemplo la University of Cambridge como editora de revistas de humanidades. Es importante el valor que cada universidad le da a su servicio editorial y sus revistas, como ejemplifica la cuarta universidad estadounidense con más revistas indexadas en WoS, la Johns Hopkins University. El valor que esta institución presta a la edición de revistas científicas aparece retratado en la iniciativa MUSE, que pretende digitalizar sus propias revistas (Lewis, 1995). Hay dos aspectos que pueden ayudar a entender el éxito de estas universidades: la colaboración con asociaciones e instituciones profesionales y la participación de proyectos editoriales agregadores, como Torch (The Online Resource Center in the Humanities); OUP's University Press Scholarship Online (UPSO), Cambridge's University Publishing Online (UPO), Jstor y Project MUSE (Hayes; Holley, 2014).

Los datos también revelan cómo las grandes editoriales universitarias, que coinciden con universidades de gran prestigio y tamaño, colaboran con asociaciones externas para la edición de revistas. La coedición de revistas es una oportunidad para la universidad, pues permite compartir costes y aumentar recursos, así como potenciar la imagen de ambas instituciones. Muchos organismos con perfiles investigadores no cuentan con editoriales propias, por lo que para tener revistas necesitan aliarse con editoriales científicas o con centros universitarios que ya tengan una. La coedición con universidades beneficia y aumenta la comunidad científica que respalda a una revista (autores, revisores, staff) y además en muchos casos se vinculan con instituciones de prestigio. Es especialmente interesante la coedición con centros con los que se colabora, como organismos de investigación públicos, asociaciones científicas o profesionales.

\section{RQ 2: Sistemas universitarios}

Según De-Moya-Anegón (2015), cuatro países (Estados Unidos, Inglaterra, Holanda y Alemania) acumulan dos tercios de las publicaciones científicas mundiales. Sin embargo, en el contexto universitario ocurre algo diferente. Reino Unido es el país con mayor número de revistas en WoS (616) seguido de Estados Unidos (455). El papel de Alemania y Holanda en la edición de revistas universitarias es insignificante, lo que implica que en estos países primen otro tipo de modelos editoriales. El peso editorial de las revistas en Latinoamérica recae en las universitarias, mientras que en otros contextos geográficos como Japón lo hace en las de asociaciones científicas (Björk, 2017). Los resultados destacan además países como Canadá, Brasil o España.

El caso concreto de España es llamativo. No existen universidades donde se concentren las revistas indexadas en WoS, pero es el cuarto país que más revistas editadas por universidades introduce en WoS. El contexto nos indica que las revistas editadas por universidades son algo más de una cuarta parte en 2007 (Abadal; Rius-Alcaraz, 2007) y una tercera parte en 2013 (Abadal; Ollé, 2013), por lo que hay una evolución positiva de la presencia de revistas editadas por uni- 
versidades. Además, si comparamos el número actual de revistas editadas por universidades españolas en WoS con el registrado en 2013 (Ruiz-Pérez; Martín-Martín; Delgado López-Cózar, 2015), nos encontramos que se ha reducido en media decena, debido a la expulsión de revistas de WoS y la adquisición de otras por parte de editoriales comerciales (Springer y Elsevier). En definitiva, las revistas españolas editadas por universidades son un componente fundamental del panorama científico nacional pero su debilidad hace que no estén carentes de riesgos, como es la expulsión de los sistemas, la adquisición por parte de otras editoriales en caso de éxito o la desaparición por la falta de relevo de los equipos editoriales (Repiso, 2019).

\section{RQ 3: Cobertura e impacto}

La presencia de las revistas editadas por universidades es contraria al tamaño de las bases de datos de WoS. En Science Citation Index, la base de datos más amplia dentro de WoS, las revistas editadas por universidades constituyen una minoría, pues apenas representan el $11,6 \%$, mientras que en la base de datos con un menor número de revistas indizadas (Arts \& Humanities Citation Index, con 1.801 revistas), la representación de éstas asciende hasta el 33,8\%. En ciencias sociales se sitúan en una posición intermedia (14,6\% del total). El estudio de Larivière sobre el monopolio de las revistas digitales indica que, en el conjunto de Web of Science, las principales editoriales comerciales, Reed-Elsevier, Wiley-Blackwell, Springer y Taylor \& Francis, están más presentes en ciencias sociales (el 70\%). En cambio, humanidades tiene menor presencia (20\%). En ciencias naturales las editoriales comerciales ocupan un puesto intermedio debido al papel relevante de algunas asociaciones científicas (Larivière; Haustein; Mongeon, 2015).

Las revistas editadas por universidades tienden a posicionarse en Science Citation Index y Social Sciences Citation Index en los últimos cuartiles. De hecho, aquellas que se encuentran en primer cuartil son mayoritariamente británicas y anglosajonas, provenientes en su mayoría de las tres universidades con más revistas (83\% de las revistas Q1) del grupo estudiadas (Oxford, Cambridge y Chicago).

Otro aspecto importante de la existencia de política editorial dentro de la universidad es su tasa de éxito, entendida por el número de revistas indexadas en WoS en comparación con aquellas editadas por el centro. Hay universidades como la Universidade de São Paulo, donde tener revistas indexadas en WoS es más una cuestión de probabilidad que de política universitaria, mientras que la University of Zagreb indiza en WoS todas las revistas que edita. La tasa de éxito es un elemento que visibiliza el interés de las universidades y la existencia de políticas activas para su desarrollo.

Dados los resultados, se plantea si tienen más interés para las universidades las revistas de ciencias sociales y humanidades que las de ciencias. La bibliografía ha mostrado que las editoriales privadas priorizan las áreas STEM (Science, Technology, Engineering and Mathematics) (Regazzi, 2015), mientras las universidades se muestran más competitivas en ciencias sociales y humanidades, al ser un nicho menos cubierto y con necesidades especiales para comunicar y difundir investigaciones. En términos relativos se aprecia la importancia de la aportación de la universidad a las humanidades y el reducido peso de sus revistas en ciencias puras. Tal y como indicaba Thatcher (2010), quizá se deba a que las editoriales universitarias editan y dan acceso a materiales académicos de calidad que no son prioritarios para los editores tradicionales.

La universidad debe repensar el valor que la edición universitaria de revistas ofrece a la institución. Tan sólo sus ventajas docentes, investigadoras y estructurales justifican la inversión y mejora del servicio editorial, el abaratamiento o incluso el acceso abierto a sus publicaciones seriadas. Las revistas son un componente importante en la marca universidad y además por su vocación internacional son un elemento público y visible, aunque en algunos casos la revista no muestre claramente que es editada por una universidad. Sin embargo una mala revista, en lugar de potenciar la marca universitaria es un perjuicio para la universidad, las ventajas se convierten en desventajas públicas cuando las universidades visibilizan un producto de baja calidad, Las editoriales privadas priorizan las áreas STEM y las universidades las ciencias sociales y humanidades, al ser un nicho menos cubierto

por su contenido o por su formato. Hace 12 años un artículo denunciaba los graves problemas formales de las webs que alojaban las revistas universitarias españolas, lo que redundaba en un mal acceso para usuarios y robots de búsqueda (Zamora et al., 2007). El éxito de una universidad de excelencia son resultados excelentes en todo lo que se proponga, y las revistas, como hemos visto, son un elemento estratégico en la marca de la universidad y por tanto en el cumplimiento de sus objetivos.

\section{Referencias}

Aaker, David A.; Álvarez-Del-Blanco, Roberto (2014). Las marcas según Aaker: 20 principios para conseguir el éxito. Barcelona: Ediciones Urano. ISBN: 9788492921072 
Aaker, David A.; Joachimsthaler, Erich (2000). Brand leadership. New York: Free Press. ISBN: 9781847398352

Abadal, Ernest; Ollé, Candela (2013). La edición universitaria en el contexto de la ciencia abierta. Barcelona: UOC. ISBN: 9788490292310

Abadal, Ernest; Rius-Alcaraz, Lluís (2008). “Revistas científicas de las universidades españolas: acciones básicas para aumentar su difusión e impacto". Revista española de documentación científica, v. 31, n. 2, pp. 242-262.

https://doi.org/10.3989/redc.2008.v31.i2.427

Ali-Choudhury, Rehnuma; Bennett, Roger; Savani, Sharmila (2009). “University marketing directors' views on the components of a university brand". International review on public and nonprofit marketing, n. 6, pp. 11-33.

https://doi.org/10.1007/s12208-008-0021-6

Björk, Bo-Christer (2017). "Journal portals - an important infrastructure for non-commercial scholarly open access publishing". Online information review, v. 41, n. 5, pp. 643-654.

https://doi.org/10.1108/OIR-03-2016-0088

Bogart, Steve (2017). SankeyMatic 2017.

http://sankeymatic.com

Carpenter, Philip (2008). "Journals, science and the future of books in humanities and social sciences". In: Conference $A$ challenge to the book in scholarship and higher education: Dodo or Dog? Amsterdam, 12-13 October.

Clement, Richard W. (2011). "Library and university press integration: A new vision for university publishing". Journal of library administration, v. 51, n. 5-6, pp. 507-528.

https://doi.org/10.1080/01930826.2011.589330

Corera-Álvarez, Elena; Molina-Molina, Silvia (2016). "La edición universitaria de revistas científicas". Revista interamericana de bibliotecología, v. 39, n. 3, pp. 277-288.

https://doi.org/10.17533/udea.rib.v39n3a05

Crane, Diane (1967). "The gatekeepers of science: Some factors affecting the selection of articles for scientific journals". The American sociologist, v. 2, n. 4, pp. 195-201.

https://www.jstor.org/stable/27701277

Cuganesan, Suresh; Petty, Richard; Finch, Nigel (2006). "Intellectual capital reporting: A user perspective". Proceedings of the Academy of Accounting and Financial Studies, v. 11, n. 1, pp. 11-17.

https://doi.org/10.2139/ssrn.902204

De-Moya-Anegón, Félix (2015). La medición de la ciencia y su importancia para la comunidad internacional. Foros Semana. El Estado de la Ciencia en Colombia. Bogotá: Semana; Colciencias.

Delgado-López-Cózar, Emilio; Fernández-Cano, Antonio (2002). "El estudio de casos en las bases de datos del Science Citation Index, Social Sciences Citation Index y Arts and Humanities Citation Index (1992-2000)". Arbor, v. 171, n. 675, pp. 609-629.

https://doi.org/10.3989/arbor.2002.i675.1049

Delgado-López-Cózar, Emilio; Ruiz-Pérez, Rafael; Jiménez-Contreras, Evaristo (2006). La edición de revistas científicas. Directrices, criterios y modelos de evaluación. Madrid: Fecyt. ISBN: 8481986976

https://www.fecyt.es/es/publicacion/la-edicion-de-revistas-cientificas-directrices-criterios-y-modelos-de-evaluacion

Esposito, Joseph J. (2007). "The wisdom of Oz: The role of the university press in scholarly communications". Journal of electronic publishing, v. 10, n. 1, pp. 1-13.

https://doi.org/10.3998/3336451.0010.103

Furlough, Michael (2008). "University presses and scholarly communication: Potential for collaboration". College \& research libraries news, v. 69, n. 1, pp. 32-36.

https://doi.org/10.5860/crln.69.1.7923

Hayes, Clayton; Holley, Robert P. (2014). "The university press: Trends, initiatives and collaborations over the past several years". Collection building, v. 33, n. 3, pp. 73-80.

https://doi.org/10.1108/CB-03-2014-0016

Larivière, Vincent; Haustein, Stefanie; Mongeon, Philippe (2015). "The oligopoly of academic publishers in the digital era". PLoS one, v. 10, n. 6, pp. 1-15.

https://doi.org/10.1371/journal.pone.0127502

Levine-Clark, Michael; Gil, Esther (2008). "A comparative analysis of social sciences citation tools". Online information review, v. 33, n. 5, pp. 986-996.

https://doi.org/10.1108/14684520911001954 
Lewis, Susan E. (1995). “Project MUSE and 'the web': An American university press goes on-line”. Logos, v. 6, n. 2, pp. 73-78.

https://brill.com/abstract/journals/logo/6/2/article-p73_5.xml

Orduña-Malea, Enrique (2012). Propuesta de un modelo de análisis redinformétrico multinivel para el estudio sistémico de las universidades españolas (2010). Valencia: Universidad Politécnica de Valencia.

http://hdl.handle.net/10251/14420

Oxford University (2018). Oxford University Press.

http://global.oup.com

Regazzi, John J. (2015). Scholarly communications: A history from content king to content as kingmakers. Maryland: Rowwman \& Littlefield. ISBN: 9780810890879

Repiso, Rafael (2019). “¿Qué valor tienen las revistas científicas para las universidades que las editan?”. En: 3a Conf intl de revistas académicas en comunicación. Universitat Pompeu Fabra: Barcelona, 15/03/2019.

https://doi.org/10.5281/zenodo.2593795

Repiso, Rafael; Chaparro-Domínguez, María-Ángeles (2018). “Universidades españolas en la prensa extranjera. Análisis de su cobertura periodística”. El profesional de la información, v. 27, n. 1, pp. 86-94.

https://doi.org/10.3145/epi.2018.ene.08

Repiso, Rafael; Merino-Arribas, Adoración; Chaparro-Domínguez, Maria-Ángeles (2016). “Agrupación de las universidades españolas en la prensa impresa nacional". Revista española de documentación científica, v. 39, n. 2, pp. 1-11. https://doi.org/10.3989/redc.2016.2.1292

Repiso, Rafael; Torres-Salinas, Daniel (2016). "Características e implicaciones de la base de datos Emerging Sources Citation Index (Thomson Reuters): las revistas en estado transitorio". Anuario ThinkEPI, v. 10, pp. 234-236.

https://doi.org/10.3145/thinkepi.2016.46

Ruiz-Pérez, Rafael; Martín-Martín, Alberto; Delgado-López-Cózar, Emilio (2015). "Las revistas universitarias en el marco de los criterios de evaluación de la actividad investigadora en España". Revista española de documentación científica, v. 38, n. 2, e081.

https://doi.org/10.3989/redc.2015.2.1191

Shapiro, Steven (2013). "JSTOR, university presses, and the serials crisis". Journal of electronic resources librarianship, v. 25, n. 3, pp. 240-242.

https://doi.org/10.1080/1941126X.2013.813319

Thatcher, Sanford G. (2010). "From the university presses. What university presses think about open access". Against the grain, v. 22, n. 3, pp. 56-58.

https://doi.org/10.7771/2380-176X.5570

Zamora, Helena; Aguillo, Isidro F.; Ortega, José-Luis; Granadino, Begoña (2007). "Calidad formal, impacto y visibilidad de las revistas electrónicas universitarias españolas". El profesional de la información, v. 16, n. 1, pp. 13-23.

https://doi.org/10.3145/epi.2007.ene.02

Zhang, Yin; Leung, Louis (2015). "A review of social networking service (SNS) research in communication journals from 2006 to 2011 ". New media \& society, v. 17, n. 7, pp. 1007-1024.

https://doi.org/10.1177/1461444813520477

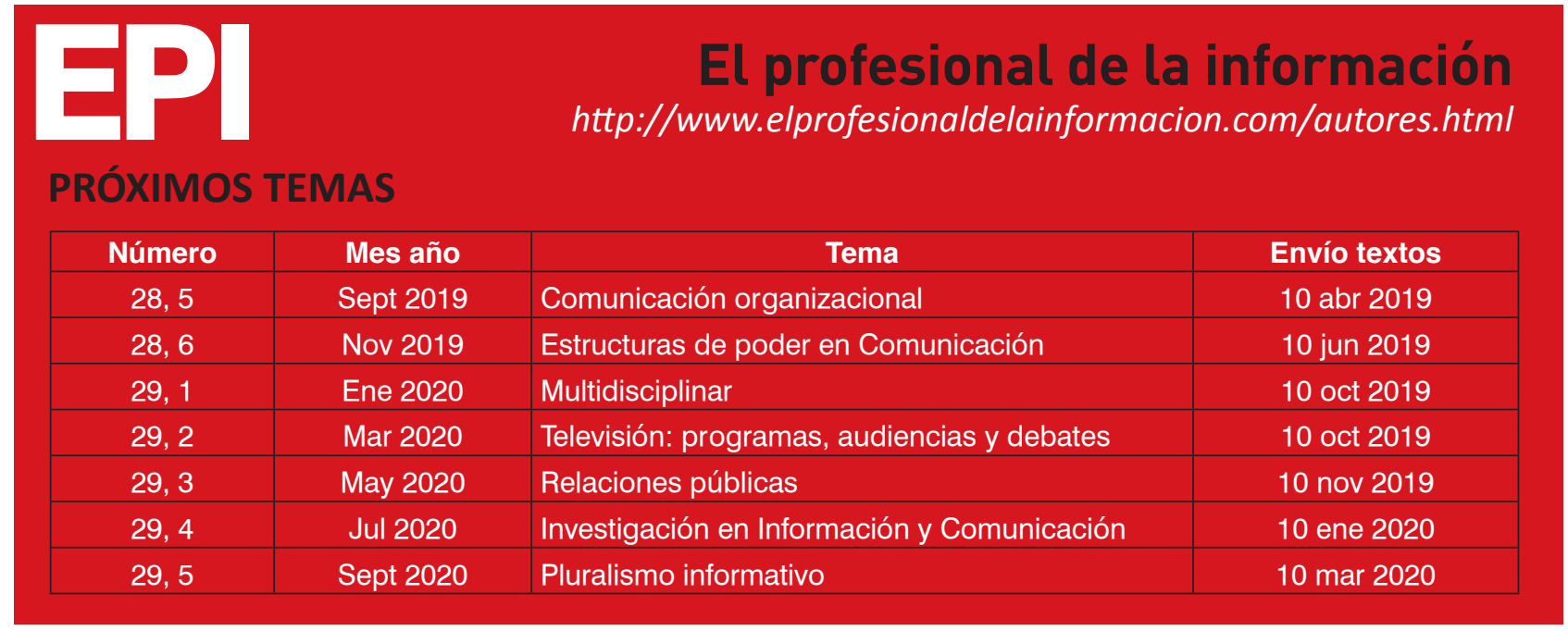

